\title{
Age-Dependent Differential Expression of Apoptotic Markers in Rat Oral Mucosa
}

\author{
Wael Youssef Elias*
}

\begin{abstract}
Objective: This study tests the hypothesis that gingival tissue produces age-dependent activation of apoptotic markers. Methods: To address the hypothesis, a prospective experimental study was conducted on 20 adult male albino rats, which were divided into two groups. Group 1 comprised rats aged six months (weighing 150-200 g), and group 2 included old rats aged one year (weighing 250-300 g). Gingival tissue and buccal mucosa biopsy samples were obtained from both groups. Histological and immunohistochemical (Bax apoptotic protein marker) sections were analyzed for both groups. Results: Our data showed a significant decrease in the proliferative activity of oral mucosa (gingiva and buccal mucosa) in old rats and an increase in the immunoreactivity of Bax apoptotic proteins related to increased susceptibility of cells to apoptosis. The mucosal structures (epithelium and lamina propria) were significantly different between the two groups. Furthermore, immunoreactivity for Bax was different between young and old rats. Conclusions: Aging is associated with changes that lead to progressive, irreversible deterioration of the functional capacities of several tissues and organs. Our study demonstrated the effect of age on the histological and apoptotic behavior of oral mucosa (gingiva and buccal mucosa) cells.
\end{abstract}

Keywords: Apoptosis- Bax- Bcl-2- gingival tissue

Asian Pac J Cancer Prev, 19 (11), 3245-3250

\section{Introduction}

Apoptosis is a process of programmed cell death that plays an important role in cell development in multicellular organisms. It helps to eliminate old or unnecessary cells without causing the release of toxic substances into the body. This well-defined sequence of events is probably the most frequent form of programmed cell death; however, other forms of cell death, which are thought to be non-apoptotic, might also be significant on the biological point of view (Leist and Jaattela, 2000). After the identification of B-cell lymphoma 2 (Bcl-2) homologs, it became clear to researchers that conserved sequence motifs or $\mathrm{BH} 1$ to $\mathrm{BH} 4$ homology domains defined a Bcl-2 family of proteins. Researchers have identified pro-survival and pro-apoptotic members in as much as 30 relatives among mammals (Borner, 2000). Besides Bcl-2, several prosurvival proteins, including Bcl-XL, Bcl-w, A1, and Mcl-1, have been identified, and these all have the domains $\mathrm{BH} 1, \mathrm{BH} 2, \mathrm{BH} 3$, and BH4. A further division of the pro-apoptotic group of Bcl-2 members includes the Bax-subfamily and the $\mathrm{BH} 3$-only proteins. The Bax-family is composed of Bax, Bak, and Bok, which all have the domains BH1, BH2, and $\mathrm{BH} 3$. In contrast, $\mathrm{BH} 3$-only proteins, comprising Bid, Bim, Bik, Bad, Bmf, Hrk, Noxa, Puma, Blk,
BNIP3, and Spike, possess only the short BH3 motif (Cory and Adams, 2002; Mund et al., 2003).

Bax undergoes major conformation changes during apoptosis and combines with the outer layer of the mitochondrial membrane and oligomerizes (Nechushtan et al., 2001). While scientists debate the mechanism, it is thought that Bax and Bak oligomers trigger or play a role in making the outer mitochondrial membrane permeable, either by the self-formation of channels (Antonsson et al., 2000) or by interacting with components of the mitochondrial pore such as voltage-dependent anion channels (Tsujimoto et al., 2000).

In most cases of human cancer, scientists have identified altered $\mathrm{Bcl}-2$ protein family expression pattern, which is thought to contribute to neoplastic cell expansion by uppressing programmed cell death and extending tumor cell life span. Scientists are still debating the function of Bcl-2 family proteins, but novel findings explain how these proteins regulate cell life and death (Kale et al., 2018). Cysteine proteases (caspases) and their activator Apaf-1 are regulated by opposing factions of the Bcl-2 protein family, whereas caspases and activators display neuronal hyperplasia, and lymphocytes and fibroblasts seem resistant to certain apoptotic stimuli (Marsden et al., 2002). Based on their structure, $\mathrm{BH} 3$ proteins are distant members of Bcl-2 proteins

Oral Diagnostic Science Department, Faculty of Dentistry, King Abdulaziz University, Jeddah, Saudi Arabia. *For Correspondence: dreliasw@gmail.com 
and genetic engineering studies have demonstrated the usefulness of these proteins in initiating programmed cell death (Puthalakath and Strasser, 2002). A genetic program activated in a dying cell regulates tissue homeostasis. Another protein, also known as Harakiri, is believed to regulate cell death. This protein triggers apoptosis and interacts selectively with Bcl-2 (Delbridge et al., 2016). Recently, it was demonstrated that the cytotoxic function of Bax relies on its ability to redistribute cytosol to the mitochondrial membrane. Furthermore, it was shown that $\mathrm{Bax}$ and $\mathrm{Bcl}-2$ could regulate cell death through interfering on independent pathways (Li et al., 2017). Bax is also known to intervene in cellular shedding or sloughing.

While the suprabasal epithelium is devoid of Bcl-2 expression, all layers, including the basal layer, show Bax protein expression. Bax increases cell susceptibility to apoptosis and may consequently affect squamous differentiation or normal epithelial cell turnover (Drenning et al., 1998). Bax positive cells have been identified in the stratum spinosum and basal part of the stratum corneum of human oral mucosa, although most cells in the stratum germinative are dedicated to dividing and differentiating terminally (Bryant and Nix, 2016).

It is necessary for unwanted cells to be removed-a process that occurs through programmed cell death. A previous study that compared programmed cell death pathways between Caenorhabditis elegans and mammals showed that the regulation of mammalian programmed cell death was complex (Richardson and Kumar, 2002). Recently developed immunohistochemical techniques demonstrated the presence of mast cells, plasma cells, and neutrophils in the Langerhans cells and macrophages in normal gingiva of mice of different ages. Investigators found a high cell density in epithelial and connective tissue, and the cell density increased significantly with age in the papillary connective tissue (Pal et al., 2016). On the other hand, Langerhans cells were detected in the epithelium, and these also varied significantly with age (Squier and Brogden, 2011). Aging is associated with a fall in many organ functions, and some evidence supports an association between advanced age and apoptotic dysregulation. A relationship has been found between age and alterations in protein levels and factors that regulate apoptosis (Joaquin and Gollapudi, 2001). The use of gene therapy and modulators of apoptosis may be potentially beneficial in preventing the functional decrease observed in the aging population (Joaquin and Gollapudi, 2001). Some researchers have suggested that apoptotic signaling varies with age, and a pro-apoptotic environment may facilitate atrophy due to aging (Suzuki et al., 2001). Some authors reported that Bax was an integral membrane protein associated with organelles or bound to organelles by Bcl-2 (Chiu et al., 2018). To determine the localization of $\mathrm{Bcl}-2$ proteins in living cells, investigators fused the green fluorescent protein (GFP) to the NH2 terminal of Bax and found that when apoptosis was induced, GFP-Bax moved intracellularly to a punctate distribution that partly localized with mitochondria. In advanced cells undergoing apoptosis, an early dramatic change was observed in the intercellular localization of Bax, and the redistribution of soluble Bax to organelles appeared to be necessary for the promotion of cell death by Bax (Chiu et al., 2018). Histological studies show that the oral mucosa is thinner and becomes narrow. In addition, as the tissue ages, keratinization decreases, and regressive changes are noted in the epithelium (Andreescu et al., 2013). Nevertheless, no significant changes in size, shape, or arrangement of epithelial cells have been related to aging. Furthermore, nutritional deficiencies such as iron or B vitamin deficiency or systemic disorders can cause histological alteration of gingival cells (Huttner et al., 2009).

The objective of this study is to test the hypothesis that gingival tissue produces age-dependent activation of apoptotic markers.

\section{Materials and Methods}

This experimental prospective study was conducted on two groups of male albino rats. The rats were selected based on animal research center recommendations and previous studies (Sherif, 2014; Wang et al., 2014; Elsharkawy and Alhazzazi, 2016; Roy et al., 2016) Thus, we selected 20 rats for each group, and each sample was prepared for about 50 histological sections. Group I consisted of 20 adult rats aged about six months and weighing between 150-200 g, whereas Group II included 20 rats aged one year weighing between 250-300 g. The animals were fed on a diet consisting of ground barley, full cream powdered milk, and fresh vegetables. The diet was supplied ad libitum. The animals were sacrificed by cervical decapitation and the buccal gingiva and mucosa of both groups were dissected out. The specimens were fixed in $10 \%$ natural buffered formalin solution, washed, and dehydrated in ascending grades of ethyl alcohol. Then the specimens were cleared in xylol and embedded in paraffin wax. Sections of the specimens measuring $5 \mu$ were subjected to routine hematoxylin and eosin staining for histological study, whereas four-micron thick sections were subjected to immunohistochemical staining. The immunohistochemical staining was a ready to use kit of the antibody against Bax antigen (Bax protein epitope specific polyclonal rabbit "GPT") that was obtained from Biogenex (Shi et al.,1991; Tacha and Chen, 1994).

\section{Results}

\section{Histology results \\ Group I Gingiva}

The gingiva of rats at six months appeared to consist of keratinized stratified squamous epithelium and underlying lamina propria (Figure 1). The epithelium ridges appeared long, numerous, irregular, variable in thickness and length, interdigitating with the papillary layer of the lamina propria. The basal cell layer (stratum basale) was composed of a single layer of columnar cells arranged perpendicularly to a well-defined basement membrane. The cells showed deeply stained oval nuclei occupying most of the cell body. The prickle or spinal cell layer (stratum spinosum) was superficial to the stratum basale, and it appeared as several rows of polyhedral shaped cells 


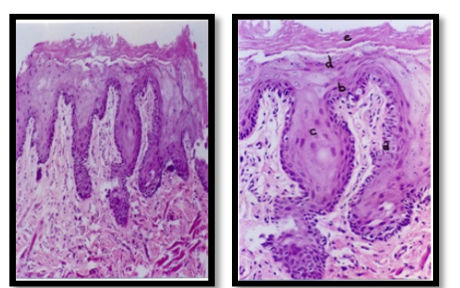

Figure 1. Representative Micrograph (Hematoxylin and eosin [H\&E] Staining, Magnification $\times 100$ ) of Group I Rat Gingiva Showing the Keratinized Stratified Squamous Epithelium, Epithelial Ridges and Lamina Propria (A) as Well as the Basal Cell Layer (a), Suprabasal Cells (b), Prickle cells (c), Granular cells (d), and Keratin Layer (e) (H\&E Staining, Magnification $\times 200)(B)$.

with centrally located rounded nuclei. The suprabasal cells of the stratum spinosum directly above the basal cell layer appeared to be formed of cells smaller than those of the superficial layers, which increased markedly in width and thickness, and their nuclei appeared vesicular and faint, with prominent nucleoli. The granular cell layer (stratum granulosum), superficial to the spinous layer, was composed of about two to three layers of flattened cells with faintly stained nuclei. The cytoplasm of these cells contained small basiophillic granules called keratohyalline granules. Finally, there was a thick acidophilic amorphous keratinized or cornified layer (stratum corneum), which appeared to be formed of keratinized squamae of the orthokeratin type.

The lamina propria was composed of a superficial papillary cell layer and deeper reticular layer. The papillary layer interdigitated with the epithelial ridges. It was composed of thin, loosely arranged collagen fibers, plenty of fibroblasts, and few blood vessels. The reticular layer appeared as dense connective tissue formed of thicker and denser fibers and poor vascularity (Figure $1 \mathrm{~A}$ and B).

\section{Buccal mucosa}

The buccal mucosa was covered by keratinized stratified squamous epithelium composed of four layers: basal cell layer (stratum basal), prickle or spinous cell layer (stratum spinosum, granular cell layer (stratum granulosum) and cornified cell layer (stratum corneum).

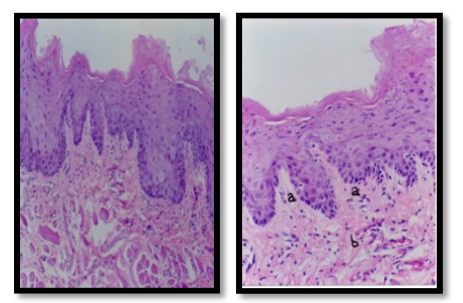

Figure 2. Representative Micrograph (Hematoxylin and eosin [H\&E] Staining, Magnification $\times 100)$ of Group II Rat Gingiva Showing Irregular Branches of Epithelium Ridges, Cornified Thick Keratin, and Coarse Collagen Bundles (H\&E staining, magnification $\times 100$ ) (A) as Well as a Thick Keratin Layer, Thick Granular Layer, Increased amount of Keratohyaline Granules and Suprabasal Wide Cells with Vesicular Nuclei Papillary Layer of Lamina Propria (a) and Blood Vessels in the Reticular layer (b) (H\&E Staining, Magnification $\times 200)$ (B).
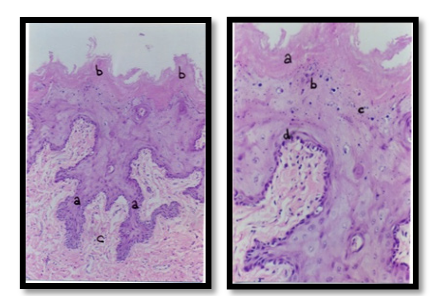

Figure 3. Representative Micrograph (Hematoxylin and eosin [H\&E] Staining, Magnification $\times 100)$ of Group II Gingiva Showing the Different Layers of Keratinized Stratified Epithelium, Epithelial Ridges (a) and Thick Keratin Layer (b), Lamina Propria(c) (A) as Well as the Keratin (a) and Different Epithelial Cell Layers (b, c, d) and Papillary Layer of the Lamina Propria (H\&E Staining, Magnification $\times 200)(B)$.

The epithelial ridges were wide, short, and numerous.

The basal cell layer (stratum basale) was composed of a single row of columnar cells arranged perpendicularly to a well-defined basement membrane. The nuclei appeared large, oval, and deeply stained, occupying most of the cell body.

The spinous or prickle cell (stratum spinosum) was composed of several rows of polyhedral shaped cells with deeply stained rounded nuclei in the suprabasal layer, while becoming faint with prominent nuclei in the superficial layers. Superficial to the stratum spinosum, was the granular cell layer (stratum granulosum), which was composed of two to three layers of flatter and wider cells with small dense deeply stained nuclei and numerous basophilic granules within the cytoplasm. The stratum corneum covering the epithelium appeared somewhat thick with flat horny scales, with no evidence of nuclei or nuclear remnant, thus appeared to be orthokeratinized.

The papillary layer appeared to have dense connective tissue fiber bundles arranged longitudinally and vertically, whereas the reticular layer fibers appeared in a meshwork pattern and more cellular and vascular than the papillary layer (Figure 2A and B).

\section{Group II Gingiva}

The gingiva appeared thicker than that of group I rats. The epithelium and lamina propria occupied a wider zone, and the cells of the stratum basal were shorter. The cells

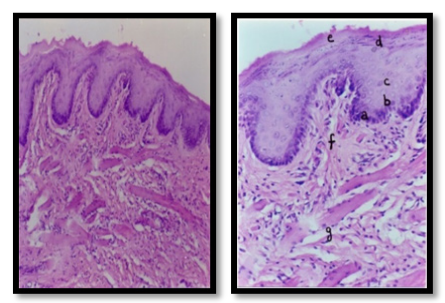

Figure 4. Representative Micrograph (Hematoxylin and eosin [H\&E] Staining, Magnification $\times 100$ ) of Group II Rat Buccal Mucosa Showing Reduced Thickness of the Epithelium and Narrow Epithelial Ridges, Thin Lamina Propria and Dense Submucosa, (A) as Well as Shrunken Basal Cells (a), Faintly Stained Suprabasal Cells (b), Large Spinous Cells with Faint and Vesicular Nuclei (c), Diminished Granular Cell Layer (d), Thin Cornified Layer (c), Reduced Lamina Propria (f), and Wide Submucosa (g), with Irregular Arrangement of Fibrous Bundles and Muscle Fibers (H\&E Staining Magnification ×200) (B). 


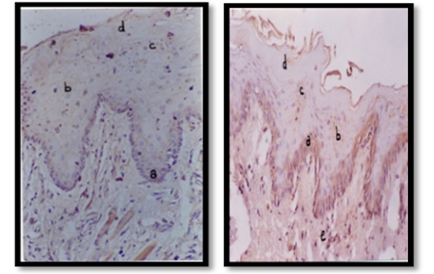

Figure 5. Representative Micrograph of Gingiva of Rats Aged Six Months Showing Mild Immunoreactivity for Bax of the Basal and Parabasal Layers (a), Mild Reaction in Localized Areas of Spinous (b), Granular (c), and Cornified Layers (d) (A). Gingiva of rats aged one year showing marked increase in immunoreactivity for Bax in the basal and parabasal layers (a), mild reaction in localized areas of the spinous (b) and granular layers (c), as well as negative reaction of the cornified layer (d). The strong reactivity is associated with the nuclei in connective tissue cells (e) (B).

of the suprabasal layer were not densely packed, and the nuclei were faint and appeared vesicular, with prominent nucleoli. The main difference existed in the stratum granulosum and stratum corneum. Regarding the stratum granulosum, there was an increase in the amount of keratohyaline granules. Furthermore, the keratin layer appeared thicker and more homogenous and cornified. The epithelial ridges appeared more irregular and branched, increasing the interface area markedly. The lamina propria appeared to have decreased cellularity and markedly increased in the fibrous component. The fibers appeared thicker, coarse and arranged in separate bundles, with poor vascularity (Figure $3 \mathrm{~A}$ and B).

\section{Buccal mucosa}

The epithelium thickness was noticeably decreased than that in the six-month-old rats (Figure 2). The cells appeared shrunken and reduced in height. However, the nuclei of the basal cell layer appeared enlarged and more deeply stained than the previous age. The supra basal cell layer corresponding to the stratum germinativum also appeared with widely enlarged faintly stained nuclei. The nuclei of the stratum spinosum appeared obviously greater in size, faint, and vesicular. The thickness of the granular cell layer was markedly diminished and consequently, the amount of keratohyaline granules was fewer than in group I rats. The cornified layer (stratum corneum) appeared thinner, flatter, and devoid of horney scales. The epithelial ridges appeared shorter and slender than those of the buccal mucosa in group I rats.

Regarding the lamina propria, it appeared narrower and more densely packed. It was coarse and composed of thick fibers with diminished vascularity than in the corresponding tissue in group I rats. Unlike in six-month-old rats, the buccal mucosa had no definite compartment and could be distinguished in the submucosal layer in one-year old rats (Figure 4A and B).

\section{Immunohistochemical results Group I Gingiva}

In six-month-old rats, the gingiva showed weak to mild immunoreactivity for Bax in the stratified squamous epithelium. The basal and parabasal layers presented mild

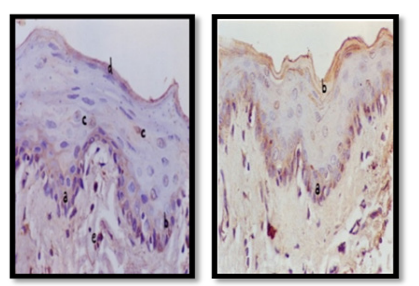

Figure 6. Buccal Mucosa of Rats Aged Six Months Showing Mild Cytosolic Reaction for Bax of Basal (a) and Parabasal Cell Layers (b), Mild Perinuclear Reaction of Few Basal Cells, Few Spinous Cells with Nuclear Reactivity (c), Mild to Moderate Reaction of the Cornified Layer (d), and Strong Perinuclear Reactivity in Few Cells of the Lamina Propria (e) (A). Buccal mucosa of rats aged one year showing increased Bax immunoreactivity in the basal and parabasal cell layers (a) and cornified layers (b) (B).

cytosolic Bax immunoreactivity of uniform staining. A mild level for Bax immunoreactivity was observed in a localized area of the cornified layer, granular and superficial spinous cells. The rest of the granular and spinous cells showed very weak reactivity, whereas the rest of the cornified layer showed negative Bax reactivity. On the other hand, the lamina propria showed mild cytosolic reactivity in the cellular components (Figure 5A).

\section{Buccal mucosa}

The majority of stratified squamous epithelial cells showed negative immunoreactivity for Bax, and only few cell layers revealed weak to mild immunoreactivity. A mild reaction was evident in the cytosol of basal and parabasal keratinocytes, and mild immunoreactivity was also associated with the nucleus of few basal cells. The spinous cells revealed mild perinuclear reaction. Additionally, the granular cell layer showed negative reactivity for Bax. Mild to moderate reactivity for Bax was identified in the thin cornified cell layer. Connective tissue cells showed moderate cytosolic immunoreactivity for Bax protein. However, strong reactivity for Bax was manifested in association with the nucleus in few apoptotic cells (Figure 6A).

\section{Group II Gingiva}

An obvious increase in the immunoreactivity for Bax was observed in group II rats compared with group I rats. The increase was substantial in the basal, parabasal, and lamina propria. However, the cornified layer was nonreactive as in group I rats except for the most superficial layer, which revealed weak to mild reaction. In addition, minute areas within the spinous and granular layers showed mild granular cytosolic Bax immunoreactivity together with few cells presenting moderate reactivity in association with their nuclei. Isolated desquamating apoptotic nuclei were identified in the cornified cells. It was also apparent that the lamina propria showed strong level for Bax reactivity, which was associated with the nuclei of most connective tissue cells, while their cytoplasm showed mild reactivity (Figure 5B) 


\section{Buccal mucosa}

Bax immunoreactivity was more noticeable in older rats. The strongest immunoreactivity appeared in the cornified layer, which showed moderate Bax reaction. A slight increase in the level of Bax was observed in the basal and suprabasal keratinocytes and was represented by mild to moderate reactivity. Conversely, a weak reaction was obvious in some spinous and granular cells. Regarding the lamina propria, no obvious changes were noticeable between group I and II rats (Figure 6B).

\section{Discussion}

This study describes the immunohistochemical distribution of Bax in normal oral mucosa of albino; it also describes the distribution of this protein marker in the gingiva and buccal mucosa of young and old rats. Bax immunostaining was mainly cytosolic and occasionally perinuclear.

In this study, oral mucosa specimens revealed changes in histological structure depending on age. In gingival tissue, increases in thickness varied as a function of age. In adult rats, the basal and parabasal cells of the gingiva were densely packed and the nuclei appeared dense, indicating a possible high mitotic division rate. However, in older rats the basal and parabasal cells were less densely packed and the nuclei in the suprabasal spinous cells were large and vesicular, and they had prominent nuclei. This might indicate a decrease in proliferative activity in these rats. Additionally, the epithelial ridges became more irregular and branching, increasing the epithelial-connective tissue interface. This indicated that the masticatory forces of rats increased by age because their masticatory muscles grow more powerful as they age. As a result, the structure of gingival mucosa depends on the nature of the rodents' diet and dentition (Huttner et al.,2009). Regarding the lamina propria, aging was associated with a decrease in cellularity and vascularity and an increase in fibrous components, with the lamina propria of the gingiva becoming coarse with increasing age-a finding that was also reported by other investigators (Andreescu et al., 2013). On the other hand, changes in the buccal mucosa, which varied with age, were detected by a decrease in the thickness of the epithelium and the cells of various layers appeared diminished and reduced in height. The cornified layer diminished in thickness and the epithelial ridges became more regular and shorter than in younger rats (Lindhe et al.,2008). Epithelial and connective tissue atrophy of the oral mucosa in older rats correlated with a decrease in vascularity and salivary gland secretion (Murayama et al., 2017).

In this study, an increase in the degree of immunostaining of Bax was associated with age. In younger rats, the gingiva showed weak reactivity for Bax. Regarding all layers of epithelium negative in the granular and cornified layers except for few localized areas. Mild cytosolic immunoreactivity for Bax was expressed in the cellular components of the lamina propria. In older rats, there was an increase in the degree of immunoreactivity, particularly in the basal and parabasal layers and lamina propria. This high immunoreactivity of
Bax in the gingival epithelium of older rats correlated with high levels of apoptosis (Andreescu et al., 2013).

On the other hand, negative immunoreactivity for Bax was observed in the buccal mucosa of younger rats except for mild cytosolic and often perinuclear involvement of the basal and parabasal layers, whereas the strongest reactivity was in the keratinous layer and underlying connective tissue lamina propria cells were similar to findings reported in human skin (Andreescu et al., 2013).

In this study, older rats showed an increase in immunoreactivity for Bax, with the strongest reaction in the cornified layer. Bax overexpression was previously reported to accelerate apoptotic cell death following a death signal (Joaquin and Gollapudi, 2001). Furthermore, Bax protein was suggested to function by itself as a cell death effector protein, and Bcl-2 might be a regulator for Bax (Li et al., 2017).

Aging is associated with decreased metabolic activity, but its effect on epithelial proliferation and rate of tissue turnover has not been confirmed (Lindhe et al.,2008). In this study, the presence of Bax in the superficial and deep layers of the epithelium suggest that despite cell loss from the surface by desquamation, cell division and apoptosis seem to occur such that constant epithelial thickness and homeostasis are maintained. Other investigators have reached the same results and found that the same genes that were involved in the regulation of cell proliferation also controlled apoptosis (Liu et al., 2017). In addition, the presence of Bax in all cell layers of the epithelium suggests that it may play a role in squamous differentiation or normal epithelial turnover by increasing the susceptibility of cells to apoptosis (Campbell and Tait, 2018).

In conclusion, our data demonstrate that apoptosis or programmed cell death is a normal physiological aging process in various tissues, including the oral mucosa. Due to aging, immunoreactivity for Bax increases in the gingiva and buccal mucosa. This increase is due to a decrease in the proliferative activity of the oral mucosa and tends to shift into maturative activity. The presence of Bax protein in all layers of the epithelium suggests that it may play role in squamous differentiation or normal epithelial turnover by increasing the susceptibility of cells to apoptosis. Thus, more studies are warranted to further elucidate the process of apoptosis and confirm that gingival tissue produces age-dependent activation of apoptotic markers. With this knowledge, the use of gene therapy and apoptosis modulators may, in the near future, have a therapeutic value in preventing the functional decline we see in the older population. Furthermore, future studies should focus on functional decline caused by dysregulation of apoptosis and how this can be prevented.

\section{References}

Andreescu CF, Mihai LL, Răescu M, et al (2013). Age influence on periodontal tissues: A histological study. Rom J Morphol Embryol, 54, 811-5.

Antonsson B, Montessuit S, Lauper S, et al (2000). Bax oligomerization is required for channel-forming activity in liposomes and to trigger cytochrome c release from 
mitochondria. Biochem $J, \mathbf{3 4 5}, 271-8$.

Borner C (2003). The Bcl-2 protein family: sensors and checkpoints for life-or-death decisions. Mol Immunol, 39, 615-47.

Bryant R, Nix D (2016). Acute and chronic wounds - E-Book Ruth Bryant, Denise Nix Google Books.

Campbell KJ, Tait SWG (2018). Targeting BCL-2 regulated apoptosis in cancer. Open Biol, 8, 180002.

Chiu WT, Chang HA, Lin YH, et al (2018). Bcl-2 regulates store-operated $\mathrm{Ca} 2+$ entry to modulate ER stress-induced apoptosis. Cell Death Dis, 4, 37.

Cory S, Adams JM (2002). The Bcl2 family: regulators of the cellular life-or-death switch. Nat Rev Cancer, 2, 647-56.

Delbridge ARD, Grabow S, Strasser A, Vaux DL (2016). Thirty years of BCL-2: translating cell death discoveries into novel cancer therapies. Nat Rev Cancer, 16, 99-109.

Elsharkawy GEZ, Alhazzazi TY (2016). The effect of the commonly used antidepressant drug amitriptyline (TCAs) on the salivary glands. $J$ Dent Oral Disord Ther, 4, 1-5.

Huttner EA, Machado DC, de Oliveira RB, Antunes AG, Hebling E (2009). Effects of human aging on periodontal tissues. Spec Care Dentist, 29, 149-55.

Joaquin AM, Gollapudi S (2001). Functional decline in aging and disease: a role of apoptosis. J Am Geriatr Soc, 49, 1234-40.

Kale J, Osterlund EJ, Andrews DW (2018). BCL-2 family proteins: changing partners in the dance towards death. Cell Death Differ, 25, 65-80.

Leist M, Jaattela M (2001). Four deaths and a funeral: from caspases to alternative mechanisms. Nat Rev Mol Cell Biol, 2, 589-98.

Li JF, Zheng SJ, Wang LL, et al (2017). Glucosylceramide synthase regulates the proliferation and apoptosis of liver cells in vitro by Bcl-2/Bax pathway. Mol Med Rep, 16, 7355-60.

Lindhe J, Lang NP, Karring T (2008). Clinical periodontology and implant dentistry, 5th edition, Blackwell Munksgaard pp 67-101.

Marsden VS, O'Connor L, O'Reilly LA, et al (2002). Apoptosis initiated by Bcl-2-regulated caspase activation independently of the cytochrome c/Apaf-1/caspase-9 apoptosome. Nature, 419, 634-7.

Liu J, Nie Z, Lei Y, Yang S, Liu Z (2017). The expression of $\mathrm{ER} \beta 2, \mathrm{Bcl}-\mathrm{xl}$ and Bax in non-small cell lung cancer and associated with prognosis 7. Int J Clin Exp Pathol, 10, 10040-6.

Mund T, Gewies A, Schoenfeld N, et al (2003). Spike, a novel BH3-only protein, regulates apoptosis at the endoplasmic reticulum. Faseb J, 17, 696-8.

Murayama K, Kawakami M, Tanaka A (2017). Chronic changes in the atrophied submandibular gland after long-term ligation of the main excretory duct in mice. J Hard Tissue Biol, 26, $13-22$.

Nechushtan A, Smith CL, Lamensdorf I, et al (2001). Bax and Bak coalesce into novel mitochondria-associated clusters during apoptosis. J Cell Biol, 153, 1265-76.

Richardson H, Kumar, S (2002). Death to flies: Drosophila as a model system to study programmed cell death. J Immunol Methods, 265, 21-38.

Roy S, Aksamitiene E, Hota S, et al (2016). Aging effects on pedicled fasciocutaneous flap survival in rats. Head Neck, 38, 1152-62.

Pal R, Shilagard T, Yang J, et al. (2016). Remodeling of the epithelial-connective tissue interface (ECTI) in oral epithelial dysplasia as visualized by noninvasive $3 \mathrm{D}$ imaging. Cancer Res, 76, 4637-47.

Puthalakat H, Strasser A (2002). Keeping killers on a tight leash: transcriptional and post-translational control of the pro-apoptotic activity of BH3-only proteins. Cell Death Differ, 9, 505-12.

Sherif IO (2014). Secoisolariciresinol diglucoside in high-fat diet and streptozotocin-induced diabetic nephropathy in rats: a possible renoprotective effect. J Physiol Biochem, 70, 961-9.

Suzuki M, Youle RJ, Tjandra N (2000). Structure of Bax: Coregulation of dimer formation and intercellular localization. Cell, 103, 645-54.

Squier C, Brogden KA (Eds.) (2011). Human oral mucosa: Squier/Human oral mucosa. West Sussex, UK: John Wiley and Sons, Ltd.

Ten Cate AR (2008). Oral histology development structure and function. Seventh edition, Elsevier, Atlanta, pp 320-75.

Tsujimoto Y, Shimizu S (2000). VDAC regulation by the Bcl-2 family of proteins. Cell Death Differ, 7, 1174-81.

Wang YP, Li G, Ma LL, et al (2014). Penehyclidine hydrochloride ameliorates renal ischemia-reperfusion injury in rats. J Surg Res, 186, 390-7.

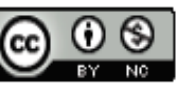

This work is licensed under a Creative Commons AttributionNon Commercial 4.0 International License. 